DRAFT VERSION JANUARY 28, 2018

Preprint typeset using $\mathrm{LAT}_{\mathrm{EX}}$ style emulateapj v. 26/01/00

\title{
FRACTIONAL AMPLITUDE OF KILOHERTZ QUASI-PERIODIC OSCILLATION FROM 4U 1728-34: EVIDENCE OF DECLINE AT HIGHER ENERGIES
}

\author{
Arunava MukherjeE ${ }^{1,2}$ And Sudip Bhattacharyya ${ }^{1}$ \\ Draft version January 28, 2018
}

\begin{abstract}
A kilohertz quasi-periodic oscillation ( $\mathrm{kHz} \mathrm{QPO})$ is an observationally robust high-frequency timing feature detected from neutron star low-mass X-ray binaries (LMXBs). This feature can be very useful to probe the superdense core matter of neutron stars, and the strong gravity regime. However, although many models exist in the literature, the physical origin of $\mathrm{kHz} \mathrm{QPO}$ is not known, and hence this feature cannot be used as a tool yet. The energy dependence of $\mathrm{kHz}$ QPO fractional rms amplitude is an important piece of the jigsaw puzzle to understand the physical origin of this timing feature. It is known that the fractional rms amplitude increases with energy at lower energies. At higher energies, the amplitude is usually believed to saturate, although this is not established. We combine tens of lower $\mathrm{kHz}$ QPOs from a neutron star LMXB 4U 1728-34 in order to improve the signal-to-noise-ratio. Consequently, we, for the first time to the best of our knowledge, find a significant and systematic decrease of the fractional rms amplitude with energy at higher photon energies. Assuming an energy spectrum model, blackbody+powerlaw, we explore if the sinusoidal variation of a single spectral parameter can reproduce the above mentioned fractional rms amplitude behavior. Our analysis suggests that the oscillation of any single blackbody parameter is favored over the oscillation of any single powerlaw parameter, in order to explain the measured amplitude behavior. We also find that the quality factor of a lower $\mathrm{kHz} \mathrm{QPO}$ does not plausibly depend on photon energy.
\end{abstract}

Subject headings: accretion, accretion disks - methods: data analysis — stars: neutron - X-rays: binaries - X-rays: individual: $4 \mathrm{U} 1728-34-\mathrm{X}$-rays: stars

\section{INTRODUCTION}

Kilohertz quasi-periodic oscillations (kHz QPOs), discovered in 1996 with Rossi X-ray Timing Explorer (RXTE; van der Klis et al. (1996); Strohmaver et al. (1996)), are the fastest variability features known till date in lowmass X-ray binaries (LMXBs). They have been observed from many neutron star LMXBs, although for a given source they are not always detected (Bhattacharyya 2010; van der Klis 2006). These QPOs often occur in pairs, and the twin peaks usually move together in the frequency range of $\sim 400-1200 \mathrm{~Hz}$. The higher frequency QPO is known as the upper $\mathrm{kHz}$ QPO (frequency $\nu_{u}$ ), and the lower frequency QPO is called the lower $\mathrm{kHz}$ QPO (frequency $\nu_{l}$ ). The frequency differences of these QPOs $\left(\Delta \nu \equiv \nu_{u}-\nu_{l}\right)$ tend to cluster around the neutron star spin frequency or half of it (van der Klis (2006) and references therein; but see Méndez and Belloni (2007)). The high frequencies of $\mathrm{kHz}$ QPOs point toward the dynamical timescale within a few Schwarzschild radii of the neutron star (Barret et al. 2005a,b, 2006; van der Klis 2006; Bhattacharvya 2010). Therefore, this observationally robust timing feature can be useful (1) to measure the neutron star parameters, which, in turn, is essential to understand the nature of super-dense degenerate matter (e.g., Bhattacharyva (2010) and references therein; Pétri (2011)); and (2) to probe the strong gravitational field regime (Psaltis 2008). However, although many models are available in the literature, the physical origin of $\mathrm{kHz}$ QPOs is still not known (e.g., Lin et al. (2011); Török (2009); van der Klis (2006)), and hence we cannot yet use this promising feature as a tool. Many proposed models for this timing feature have attempted to explain the frequencies (Miller et al. 1998; Stella and Vietri 1998, 1999; Lamb and Miller 2003; Kluzniak \& Abramowicz 2001; Abramowicz and Kluźniak 2001; Wiinands et al. 2003; Lee et al. 2004; Zhang 2004; Zhang et al. 2006; Mukhopadhvay 2009; Alpar and Psaltis 2008; Stuchlík et al. 2011). Some of these models involve several general relativistic frequencies at preferred radii and the neutron star spin frequency; in some cases beating and/or resonances among these frequencies. Other models include the association of $\mathrm{kHz}$ QPOs with accretion through a non-axisymmetric magnetic boundary layer in the unstable regime (Romanova and Kulkarni 2009); attempts to connect the $\mathrm{kHz}$ QPOs with the trapped twoarmed nearly vertical oscillations in vertically isothermal disks with toroidal magnetic fields (Kato 2011); attribution of the upper $\mathrm{kHz}$ QPO profile to the radial extent of the $\mathrm{kHz}$ QPO emission region associated with the transitional layer at the magnetosphere-disk boundary (Wang et al. 2011), etc.

However, modeling only the frequencies gives an incomplete picture; probing radiative transfer and further modulation processes is essential to constrain the existing models for understanding the physical mechanism giving rise to $\mathrm{kHz}$ QPOs. For example, the models based on frequencies tentatively suggest some locations of $\mathrm{kHz} \mathrm{QPO}$ origin. These locations could be at certain radii of the accretion disk, such as the innermost-stable-circular-orbit radius, sonic point radius, etc. (see van der Klis (2006) and refer-

\footnotetext{
${ }^{1}$ Tata Institute of Fundamental Research, Mumbai-400005, India; arunava@tifr.res.in

${ }^{2}$ Inter-University Centre for Astronomy and Astrophysics, Pune, India; arunava@iucaa.ernet.in
} 
ences therein). However, one needs to independently verify these proposed locations. Since different energy spectral components originate from different locations, such as disk, boundary layer, corona, etc., a connection found between a $\mathrm{kHz}$ QPO property and a spectral component could provide this independent verification. Therefore, it is essential to study the energy dependence of $\mathrm{kHz} \mathrm{QPO}$ properties.

In this paper, we study the energy dependence of fractional rms amplitude of $\mathrm{kHz}$ QPOs. This property is a measure of QPO strength, and hence studying energy dependent $\mathrm{kHz}$ QPO property can be useful to probe which spectral component primarily contributes to this timing feature. This spectral connection of $\mathrm{kHz}$ QPO has been discussed by some authors. For example, Méndez (2006) suggested that, while the $\mathrm{kHz}$ QPO frequencies are plausibly determined at the disk, this feature is modulated at the high-energy spectral component (e.g., corona, boundary layer, etc.). It has been reported by several authors that the $\mathrm{kHz}$ QPO fractional rms amplitude increases with photon energy at lower energies, and then plausibly saturates (van der Klis (2006) and references therein; Gilfanov et al. (2003)). Several authors have theoretically computed the energy dependence of rms amplitude of Comptonizing component variability (e.g., Cabanac et al. (2010); Gierliński \& Zdziarski (2005); Zdziarski et al. (2005); Lee and Miller (1998)). For example, Cabanac et al. (2010) have demonstrated that oscillating hot thermal corona may give rise to an overall increase in rms variability with photon energy. However, the above mentioned saturation at higher energies is not observationally established for many sources due to the lack of sufficient signal-to-noise ratios $(\mathrm{S} / \mathrm{Ns})$ at higher energies. For example, while the higher energy data points of Fig. 10 of Gilfanov et al. (2003) are consistent with a flat fractional rms amplitude versus energy behavior, the errors of these data points are quite high.

With this motivation, we try to measure the fractional rms amplitude versus energy behavior of lower $\mathrm{kHz}$ QPOs with improved S/N. We combine tens of lower $\mathrm{kHz}$ QPOs from the neutron star LMXB 4U 1728-34, in order to improve the S/N (e.g., Ford and van der Klis (1998); Méndez and van der Klis (1999); Di Salvo et al. (2001)). We choose lower $\mathrm{kHz}$ QPO, because this narrow QPO is more frequent and easier to detect than the relatively broad and weak upper $\mathrm{kHz}$ QPO. We find that the fractional rms amplitude systematically decreases with energy at higher energies. In order to understand this new finding, we compare the data with models involving a blackbody+powerlaw energy spectrum. This comparison suggests that the observed behavior of fractional rms amplitude may be reproduced with a fluctuation of the blackbody spectral component. In addition, we find that the quality factor (Q) of lower $\mathrm{kHz} \mathrm{QPO}$ does not plausibly depend on energy.

In $\S 2$ and $\S$, we describe our data analysis technique in detail and display our results, respectively. In $\S$, we describe the models and discuss which ones are favored. Finally, in $\S$ 5, we summarize our results and give implications.

\section{DATA ANALYSIS}

In order to study the energy dependence of fractional rms amplitude associated with lower $\mathrm{kHz}$ QPOs, we retrieved all $R X T E$ proportional counter array (PCA) pointed observational data of the source $4 \mathrm{U}$ 1728-34 for the period of April 13, 2000 to the end of December, 2009 $(\approx 272 \mathrm{ks}$ of cleaned exposure time) corresponding to the PCA gain epoch 5. Note that gain, and hence the energychannel conversion 3 , has changed during the entire $R X T E$ lifetime. Therefore, we restrict our analysis to the data of a single epoch in order to make the energy dependence measurement more reliable. Although even within an epoch the energy-channel conversion evolves, such changes would be negligibly small to significantly affect the final conclusion of the analysis. We choose epoch 5 , because it has the largest amount of data among all the epochs. We consider only the science event files with time resolution $\sim 122 \mu$ s and having continuous exposure time of at least $400 \mathrm{~s}$ for searching $\mathrm{kHz}$ QPOs. We do not apply any filtering based on energies, PCUs, Xenon layers, etc. However, following the usual practice, we filter the data based on time in order to remove thermonuclear X-ray bursts, data gaps, and observed intensity increase/decrease due to instrumental effects (especially due to start or stop of a PCU).

It has been observed that all the $\mathrm{kHz}$ QPOs (which are strong and narrow) are confined to a small portion, i.e., lower banana, of the color-color diagram (CD) of 4U 172834 (Di Salvo et al. 2001). This suggests that they are of the same spectral origin. The strong inter-dependence found between Q-values and frequencies (Barret et al. 2006) of lower $\mathrm{kHz}$ QPOs from $4 \mathrm{U}$ 1728-34 suggests that all the QPOs considered by us are of same physical origin. These justify the combination of many $\mathrm{kHz}$ QPOs (as mentioned in $\S$ 1).

We follow a few steps to probe the energy dependence of lower $\mathrm{kHz}$ QPO amplitudes. In the first step, we collect all the significant $\mathrm{kHz}$ QPOs from the event files (with at least $400 \mathrm{~s}$ of data) using a blind search. In order to do this, we compute a Leahy-normalized (Leahy et al. 1983) power density spectrum (PDS) using discrete Fourier transform (DFT) 4 from each $10 \mathrm{~s}$ of data for a given event file van der Klis 1989). The Nyquist frequency and frequency resolution of each such PDS (from $10 \mathrm{~s}$ of data) are $2048 \mathrm{~Hz}$ and $0.1 \mathrm{~Hz}$, respectively (van der Klis 1989). Then all (say, $N$ ) the PDSs from a given event file are averaged, and we search for $\mathrm{kHz}$ QPOs in the frequency range of $400-1400 \mathrm{~Hz}$ in this mean PDS. In order to search effectively, several (say, $W$ ) adjacent frequency bins are combined. The noise powers in this PDS follow a $\chi^{2}$ distribution with $2 N W$ degrees of freedom (van der Klis 1989). For a putative peak, we compute the single trial probability $(q)$ of occurrence for a power greater than or equal to the peak power by chance from the noise power distribution. Note that we consider the peak power, and do not fit the peak with a function (say, Lorentzian) for significance calculation. The probability $q$ is multiplied with the number of trials $\left(N_{\text {trial }}\right)$ in order to obtain the final probability $\epsilon\left(=q N_{\text {trial }}\right)$. We use $N_{\text {trial }}=20480$, i.e., the original total number of frequency bins in a mean PDS. We consider a putative peak as a $\mathrm{kHz} \mathrm{QPO}$, if $\epsilon \leq 4.65 \times 10^{-4}$ (i.e., at

${ }^{3}$ http://heasarc.gsfc.nasa.gov/docs/xte/e-c_table.html

${ }^{4}$ DFT follows the same statistics of Leahy-normalization (van der Klis 1989). 
least $3.5 \sigma$ significant). We detect tens of $\mathrm{kHz}$ QPOs from $4 \mathrm{U} 1728-34$ using the above procedure.

In the second step, described in this paragraph, we identify the lower $\mathrm{kHz}$ QPOs from the QPOs detected using the above mentioned criterion. If twin $\mathrm{kHz} \mathrm{QPO}$ peaks appear in the mean PDS from an event file, the identification of the lower $\mathrm{kHz}$ QPO requires no further effort. If there is only one peak for an event file, then we consider it as a lower $\mathrm{kHz} \mathrm{QPO}$, if its $Q \geq 40$. This is because, the $\mathrm{Q}$ vs frequency diagram of $4 \mathrm{U}$ 1728-34 (Barret et al. 2006) clearly shows that only the lower $\mathrm{kHz}$ QPOs can have Qvalues greater than 40 , and all the upper $\mathrm{kHz}$ QPOs have smaller Q-values. In order to estimate the Q-values of $\mathrm{kHz}$ QPOs, we first use the shift-and-add technique (e.g., Méndez et al. (1998); Barret et al. (2006)) within each event file considering the entire PCA energy range. This technique mostly corrects for the centroid frequency drift. Note that such uncorrected drift makes the measured Qvalue smaller than the actual value. After application of shift-and-add, we fit each PDS containing a detected $\mathrm{kHz}$ QPO with a 'constant + powerlaw + Lorentzian' model; constant takes care of the Poissonian white noise, powerlaw takes care of the low frequency red noise, and a Lorentzian describes the $\mathrm{kHz}$ QPO. We obtain the best-fit centroid frequency $(\nu)$ and the best-fit full width at half maximum (FWHM) from the Lorentzian component, and compute the Q-value $(\nu / \mathrm{FWHM})$. Then we identify the lower $\mathrm{kHz}$ QPOs using the criterion $Q \geq 40$. From the entire $\approx 272 \mathrm{ks}$ of data, 40 lower $\mathrm{kHz}$ QPOs (each from one event file) are identified spanning $86.32 \mathrm{ks}$ of exposure time, and are considered for further analysis.

These 40 lower $\mathrm{kHz}$ QPOs are now to be combined in order to study the energy dependence of fractional rms amplitude with improved S/N. However, before doing this, we perform a few preliminary analyses in the third step, described in this paragraph. In this step, we still use the entire PCA energy range. We consider non-overlapping 400-second segments from each of 40 event files with a lower $\mathrm{kHz}$ QPO. Now we collect only those 400-second segments, in each of which a lower $\mathrm{kHz}$ QPO peak with $\epsilon \leq 2.7 \times 10^{-3}$ (corresponding to $3 \sigma$ ) exists. To calculate the $\epsilon$ for a given 400-second segment, we further divide the segment into 40 segments of 10 -second intervals, calculate PDS for each 10-second interval, average these 40 PDSs, and use $N=40$ and $N_{\text {trial }}=10000 / W$. Note that this procedure, as well as the definitions of $\epsilon, N, W$ and $N_{\text {trial }}$ are given in the third paragraph of the current section. We find 173 number of 400-second segments with strong lower $\mathrm{kHz}$ QPO peaks using the above mentioned criterion on $\epsilon$. The mean of these peaks is $\nu_{\text {mean }}=807.8 \mathrm{~Hz}$. We also calculate $\nu_{\text {diff }}$, which is the separation between $\nu_{\text {mean }}$ and the centroid frequency of an individual lower $\mathrm{kHz} \mathrm{QPO}$, for each 400-second segment. We use these 173 segments, $\nu_{\text {mean }}$, and $\nu_{\text {diff }}$ values for further analysis.

In the fourth step, described in this and the next three paragraphs, we compute lower $\mathrm{kHz} \mathrm{QPO}$ fractional rms amplitudes for several chosen energy ranges after combining the data of 173 number of 400-second segments (see the previous paragraph). We consider a set of PCA absolute channel ranges $5-8,9-11,12-13,14-15,16-17,18-21,20-$ $25,22-25,22-29,24-31,26-33$ and $26-49$ corresponding to the energy ranges $2.06-3.68,3.68-4.90,4.90-5.71,5.71-$ $6.53,6.53-7.35,7.35-8.98,8.17-10.63,8.98-10.63,8.98-$ $12.28,9.81-13.11,10.63-13.93$ and $10.63-20.62 \mathrm{keV}$, respectively 5 , in order to study the energy dependence of fractional rms amplitude. We do not use proportional counter unit 0 (PCU0) in this step, because the energychannel conversion for PCU0 is somewhat different from that of the other four PCUs during epoch 5. For each energy range, we compute a mean Leahy-normalized PDS (in the same way as described earlier in this section) for each of 173 number of 400-second segments. Then, we shift each of 173 lower $\mathrm{kHz}$ QPO peaks by $\nu_{\text {diff }}$ (the value for the entire PCA energy range; see the previous paragraph) to align them at $\nu_{\text {mean }}$, and add them together to obtain a grand average Leahy-normalized PDS for every energy range.

Now the question is whether the centroid frequencies of the $\mathrm{kHz}$ QPOs change with energy. Such a change might affect our grand average Leahy-normalized PDS and further results. We cannot check it directly for individual $\mathrm{kHz}$ QPOs, because the statistics is not often adequate to detect an individual $\mathrm{kHz} \mathrm{QPO}$ in a small energy range. So we check it in the following way. If we consider that centroid frequency of each of the individual $\mathrm{kHz}$ QPOs is energy dependent, then there are two extreme possibilities. (1) The first possibility: the centroid frequencies of all these $\mathrm{kHz}$ QPOs either increase or decrease at higher energy in a similar way. In this case, the $\nu_{\text {mean }}$ value is also expected to either increase or decrease at higher energy, since we do not recalculate $\nu_{\text {diff }}$ separately for each energy range. But we find that the $\nu_{\text {mean }}$ value remains the same in all the energy bands (Figs. 1 and 22). (2) The second possibility: the centroid frequencies of some $\mathrm{kHz}$ QPOs increase, and those of some other decrease with the increase of energy, in such a way that all the $\nu_{\text {mean }}$ values at various energy ranges remain the same. In this case, although the centroid frequency would not change, the width of the merged kHz QPOs (i.e., after applying the shift-and-add method) is expected to systematically increase resulting in a lower and lower Q-value, at higher and higher energies. But we find that all the measured Q-values are quite close to each other (within the errors) and no systematic variation is observed. The fact that both $\nu_{\text {mean }}$ values and Q-values remain quite similar in all the energy ranges (Figs. 1, 2, and 3) suggests that the centroid frequencies of the $\mathrm{kHz}$ QPOs do not change with energy.

The shift-and-add technique to obtain a grand average Leahy-normalized PDS (mentioned above) is a standard method to improve the $\mathrm{S} / \mathrm{N}$ of $\mathrm{kHz} \mathrm{QPOs}$ and to correct their Q-value (e.g., Barret et al. (2006); Méndez (2006); Méndez et al. (2001); Méndez and van der Klis (1999); Méndez et al. (1998); Mukherjee and Bhattacharyya $(2011 \mathrm{~b}))$. The grand average Leahy-normalized PDS for each energy range is then fitted with a 'constant + powerlaw + Lorentzian' model (as before), and the fractional rms amplitude and the Q-value are computed from the best-fit Lorentzian parameters (see the equation 4.10 of van der Klis (1989) for a general rms amplitude formula).

In the fifth step, we correct the above mentioned fractional rms amplitudes for the background levels. First, we compute the background count rate in each of the chosen

\footnotetext{
${ }^{5}$ http://heasarc.gsfc.nasa.gov/docs/xte/e-c_table.html
} 
Mukherjee and Bhattacharyya

energy ranges using the appropriate channel range of the corresponding standard-2 data files. In order to do this, we use the PCA background model file for bright sources, and the 'FTOOLS' command 'pcabackest'. Then, we compute the background corrected fractional rms amplitudes $\left(R_{c}\right)$ from the uncorrected fractional rms amplitudes $\left(R_{u c}\right)$, the total count rates $(I)$ and the background count rates $(B)$ using the formula $R_{c}=R_{u c} \times I /(I-B)$ (Muno et al. 2002).

Apart from the energy dependence of fractional rms amplitude, we estimate how the Q-value of the lower $\mathrm{kHz}$ QPO depends on energy. This is computed from the above mentioned fitting of the grand average Leahy-normalized PDS for each energy range. The ratio of the best-fit centroid frequency to the best-fit FWHM of the Lorentzian model component gives the Q-value. Here we note that while the shift-and-add technique corrects for a large error in Q-value due to the centroid frequency drift, this technique also introduces a small error in Q-value, because the centroid frequency is shifted without changing the FWHM.

\section{RESULTS}

The frequencies of the 173 detected lower $\mathrm{kHz}$ QPOs, which are used to study the energy-dependence of fractional rms amplitude ( $(2)$, span the range of $669.4-912.5$ $\mathrm{Hz}\left(\right.$ mean $\left(\nu_{\text {mean }}\right)=807.8 \mathrm{~Hz}$; median $=810.2 \mathrm{~Hz}$, and standard deviation $=57.8 \mathrm{~Hz}$ ). In Figs. 1 and 2 , the grand average Leahy-normalized PDSs (see $\S$ 2) for 12 energy ranges are shown in 12 panels. Each panel is for the same exposure $(69.2 \mathrm{ks})$, and in each panel the total count rate and the background count rate are mentioned. These figures indicate that, while there are visibly prominent $\mathrm{kHz}$ QPO peaks for the lower energy bands, such a peak is not visible for the highest energy band.

In Fig. 3, we plot background corrected fractional rms amplitude versus energy. The amplitude increases with energy at lower energies, and systematically decreases (seen from the overlapping energy ranges) above $\approx 10 \mathrm{keV}$. Note that, if for an energy range the lower $\mathrm{kHz} \mathrm{QPO}$ peak is not clearly visible (see Fig. 2. panels with blue model curves), we compute an upper limit to the fractional rms amplitude at $\nu_{\text {mean }}($ see $\S 2$ 2) instead of estimating the amplitude from the best-fit Lorentzian. Our estimated $1 \sigma$ and $3 \sigma$ upper limits of fractional rms amplitude in the energy range $10.63-20.62 \mathrm{keV}$ are $2.37 \%$ and $3.66 \%$, respectively. If we compare the fractional $\mathrm{rms}$ amplitude value $(=14.38 \% \pm 1.71 \%)$ in the $7.35-8.98 \mathrm{keV}$ range with the $3 \sigma$ upper limit $(=3.66 \%)$ in the $10.63-20.62 \mathrm{keV}$ range, we find a $6.3 \sigma$ significant drop in the amplitude value. This quantifies the decrease of the lower $\mathrm{kHz} \mathrm{QPO}$ fractional rms amplitude at higher energies (see Fig. 3).

The initial increasing part of the fractional rms amplitude is fitted with a linear and a powerlaw model, the best-fit curves of which are very similar to each other (see Fig. (4). This figure, with extrapolated model curves, shows that the increasing trend of lower $\mathrm{kHz}$ QPO amplitude at lower energies cannot plausibly explain the upper limit in the $10.63-20.62 \mathrm{keV}$ range. For example, the extrapolated fractional rms amplitude value at 10.63-20.62 $\mathrm{keV}$ from the linear model is $30.08 \pm 6.80$, which implies a $3.89 \sigma$ significant higher value compared to the $3 \sigma$ upper limit of the observed fractional rms amplitude.

While the fractional rms amplitude changes with energy,
Q-value does not (Fig. 3. lower panel). As mentioned in $\S 2$, while the shift-and-add technique corrects for a usually large error in Q-value due to the evolution of the centroid frequency, this technique introduces a small error. This is because, while a $\mathrm{kHz}$ QPO peak is shifted to a new frequency $\left(\nu_{\text {mean }}=807.8 \mathrm{~Hz}\right)$, the FWHM of this peak remains the same. Given that the standard deviation of the centroid frequency distribution of our detected lower $\mathrm{kHz}$ QPOs is $57.8 \mathrm{~Hz}$ and $\nu_{\text {mean }}=807.8 \mathrm{~Hz}$, this error can be estimated to be $\sim 7 \%$ of the typical value of $\mathrm{Q} \sim 80$. Therefore, this error is small compared to the $1 \sigma$ error (which is typically $\sim 25 \%-45 \%$ ) from Lorentzian fitting of the QPO peaks. Therefore, the error due to frequency shift does not change our conclusion regarding the energy independence of Q-values, and hence we do not make an attempt to correct it.

\section{COMPARISON WITH MODELS}

This paper shows a prominent and systematic decrease of the lower $\mathrm{kHz}$ QPO fractional rms amplitude at higher energies, for the first time to the best of our knowledge. We, therefore, try to find out if such a decrease is at all theoretical expected. For example, could certain parameter values of the usual energy spectra of neutron star LMXBs explain it qualitatively? Before testing this, let us briefly mention the X-ray components of these sources, as we understand currently. Neutron star LMXBs are believed to have two primary X-ray emitting regions, an accretion disk and a boundary layer. Both these regions are expected to be optically thick, and therefore to emit blackbody radiation. Furthermore, one (or more) of these components may be covered with a corona (coronae). Such a corona may reprocess (Comptonize) the blackbody photons. The amount of reprocessing depends on the optical depth of the corona, as well as the extent of coverage (e.g., full versus partial). As a result, the observed spectrum either from the disk and/or the boundary layer can be a blackbody, or Comptonized, or a sum of both. Observationally, however, no spectral model usually uniquely describes the data. Moreover, it is not usually clear, where various components of a given model originate from. Therefore, although many works over a few decades (e.g., Mitsuda et al. (1989); Mitsuda and Dotani (1989); White et al. (1988); $\quad$ Church and Balucińska-Church (2001); Christian and Swank (1997); Maccarone and Coppi (2003); Maitra and Bailvn (2004); Gilfanov et al. (2003); Olive et al. (2003); Wiinands (2001); Barret (2001): Lin et al. (2007, 2010); Mukherjee and Bhattacharyya (2011a)) support the general understanding mentioned above, the details are still unknown.

In order to have a basic understanding (i.e., as much as possible independent of detailed models) of the lower $\mathrm{kHz} \mathrm{QPO}$ fractional rms amplitude versus energy behavior found by us, we choose the simplest two-component spectral model, which is based on the above mentioned description of X-ray components. This is a blackbody+powerlaw model, in which powerlaw usually represents the Comptonized component. Tarana et al. (2011) and Seifina and Titarchuk (2011) have successfully fitted the continuum spectra from $4 \mathrm{U} 1728-34$ with a blackbody+Comptonization model. In order to check whether a blackbody+powerlaw works, we fit the continuum spectra of a few data files (having prominent $\mathrm{kHz}$ QPOs) with 
this model, as well as with a blackbody+Comptonization (bbodyrad+compTT in XSPEC) model. We find that, not only both the models give acceptable fits, but the powerlaw component represents the Comptonization component reasonably well, as verified from the respective fluxes and spectral component curves. This indicates that a blackbody+powerlaw model is appropriate for the $4 \mathrm{U}$ 1728-34 spectra.

Before describing our model, here we mention and discuss the assumptions involved in our modeling and interpretation, some of them recapitulated from the data analysis side and some other extra assumptions from the model side. (1) We assume that the spread in energy spectra (e.g., soft colors and hard colors in a color-color diagram (CD); van der Klis (2006)) and in intensity from our data files containing lower $\mathrm{kHz}$ QPOs do not significantly affect the robustness of the energy behavior of fractional rms amplitude reported by us (Fig. 31). This is justified because of the following reasons. (a) All the QPOs (which are strong and narrow) considered by us are confined to a small portion, i.e., lower banana, of the $\mathrm{CD}$ of $4 \mathrm{U} 1728$ 34 (Di Salvo et al. 2001), as mentioned in $\S 2$ (b) Given the small fractional rms amplitudes $(\sim 5-14 \%)$ of lower $\mathrm{kHz}$ QPOs in the $2-16 \mathrm{keV}$ energy range, which is used to compute the $\mathrm{CD}$ and HID, the entire X-ray energy spectrum does not contribute to these QPOs. Hence a small spread in CD does not necessarily indicate different physical origin of different lower $\mathrm{kHz}$ QPOs. (c) The strong inter-dependence found between $\mathrm{Q}$-values and frequencies of lower $\mathrm{kHz}$ QPOs from $4 \mathrm{U}$ 1728-34 (along with several other atoll sources; Barret et al. (2006)) suggests that all the QPOs considered by us are of same physical origin. These support the robustness of our reported rms-energy behavior. (2) We assume that the energy dependence of lower $\mathrm{kHz}$ QPOs does not change with frequency. This may be a reasonable assumption, because these QPOs with different frequencies are likely to have originated from the same physical process. (3) Our model considers the sinusoidal fluctuation of a single parameter of the above mentioned blackbody+powerlaw model. The sinusoidal fluctuation is somewhat justified, because according to some models, the original oscillations are sinusoidal, and the broadening happens because of a decoherence mechanism (say, damping; van der Klis (2006) and references therein). Here we note that a sinusoidal signal was also assumed by Lee and Miller (1998). We further note that, even if the intrinsic oscillations of lower $\mathrm{kHz}$ QPOs are a sum of many sinusoids originated separately, our modeling will still be useful to connect a spectral component to these QPOs. (4) Our model does not involve any response matrix. Although application of a response matrix may slightly change the model rms-energy curves quantitatively, the qualitative nature of these curves should not change.

Now we describe our model. As previously mentioned, the total time averaged flux (say, $\langle S(E, t)\rangle ; E$ : photon energy, $t$ : time) of the source can be fitted well with a combination of blackbody $\left(S_{\mathrm{BB}}\right)$ and powerlaw $\left(S_{\mathrm{PL}}\right)$. Each of blackbody and powerlaw has two parameters: normalization $\left(N_{\mathrm{BB}}\right)$ and temperature $\left(T_{\mathrm{BB}}\right)$ for the former, and normalization $\left(N_{\mathrm{PL}}\right)$ and photon index $\left(\alpha_{\mathrm{PL}}\right)$ for the latter. We try to investigate if sinusoidal fluctuation in one of these parameters (while keeping the other three parameters non-fluctuating) can reproduce the observed fractional rms amplitude versus energy behavior. Here as an example, we consider the case of fluctuating blackbody temperature.

$$
\begin{aligned}
N_{\mathrm{H}} \times S(E, t) & =N_{\mathrm{H}} \times\left[S_{\mathrm{BB}}\left(E, N_{\mathrm{BB}}, T_{\mathrm{BB}}(t)\right)\right. \\
& \left.+S_{\mathrm{PL}}\left(E, N_{\mathrm{PL}}, \alpha_{\mathrm{PL}}\right)\right] \\
\text { where, } T_{\mathrm{BB}}(t) & =T_{\mathrm{BB} 0}+T_{\mathrm{BB} 1} \operatorname{Sin}(\omega t) \\
N_{\mathrm{H}} \times<S(E, t)> & =N_{\mathrm{H}} \times\left[<S_{\mathrm{BB}}\left(E, N_{\mathrm{BB}},\left(T_{\mathrm{BB} 0}\right.\right.\right. \\
& \left.\left.+T_{\mathrm{BB} 1} \operatorname{Sin}(\omega t)\right)>+S_{\mathrm{PL}}\left(E, N_{\mathrm{PL}}, \alpha_{\mathrm{PL}}\right)\right] \\
N_{\mathrm{H}} \times \sigma_{S}\left(=\sigma_{N S}\right) & =N_{\mathrm{H}} \times \sqrt{ }\left(<S(E, t)^{2}>-<S(E, t)>^{2}\right) \\
\text { fracRMS } & =\frac{\sigma_{N S}}{N_{\mathrm{H}} \times<S(E, t)>} \\
& =\frac{\sqrt{ }\left(<S(E, t)^{2}>-<S(E, t)>^{2}\right)}{<S(E, t)>}
\end{aligned}
$$

which is independent of $N_{\mathrm{H}}$ (neutral hydrogen column density). Here, fracRMS is our model fractional rms amplitude. Similarly, we compute the model fractional rms amplitude in each case when any one of the other three parameters varies sinusoidally in time.

We note that the blackbody flux and the powerlaw flux are nonlinear functions of $T_{\mathrm{BB}}$ and $\alpha_{\mathrm{PL}}$, respectively. Therefore, if $T_{\mathrm{BB}}$ (or $\alpha_{\mathrm{PL}}$ ) fluctuates sinusoidally (as we assume), the variation of the blackbody flux (or powerlaw flux) will not be strictly sinusoidal, and will contain higher harmonics. Here we show that this flux variation is sinusoidal (which we assume in our modeling) when $T_{\mathrm{BB}}$ (or $\alpha_{\mathrm{PL}}$ ) fluctuation is small (e.g., $T_{\mathrm{BB} 1} \ll T_{\mathrm{BB} 0}$, for the varying blackbody temperature), and is therefore considered up to the first order:

$$
\begin{aligned}
<S(E, t)>= & <S_{\mathrm{BB}}\left(E, N_{\mathrm{BB}},\left(T_{\mathrm{BB} 0}+T_{\mathrm{BB} 1} \operatorname{Sin}(\omega t)\right)\right)> \\
+ & <S_{\mathrm{PL}}\left(E, N_{\mathrm{PL}}, \alpha_{\mathrm{PL}}\right)> \\
= & <S_{\mathrm{BB}}\left(E, N_{\mathrm{BB}}, T_{\mathrm{BB} 0}\right)> \\
+ & <S_{\mathrm{BB} 1}\left(E, N_{\mathrm{BB}}, T_{\mathrm{BB} 0}, \mathrm{~T}_{\mathrm{BB} 1}\right) \operatorname{Sin}(\omega t)> \\
+ & O\left[\frac{T_{\mathrm{BB} 1}}{T_{\mathrm{BB} 0}}\right]^{2}+S_{\mathrm{PL}}\left(E, N_{\mathrm{PL}}, \alpha_{\mathrm{PL}}\right) \\
= & S_{\mathrm{BB}}\left(E, N_{\mathrm{BB}}, T_{\mathrm{BB} 0}\right)+S_{\mathrm{PL}}\left(E, N_{\mathrm{PL}}, \alpha_{\mathrm{PL}}\right) \\
& \left(\text { which is correct up to first order in }\left[\frac{T_{\mathrm{BB} 1}}{T_{\mathrm{BB} 0}}\right]\right)
\end{aligned}
$$

The last step is justified because the observations are several orders of magnitudes longer than the oscillation pe$\operatorname{riod} T=2 \pi / \omega$. This last step shows that, $\langle S(E, t)\rangle$ $=S(E)$; which is simply the sum of XSPEC model components (bbodyrad+powerlaw) used to fit the time averaged spectrum. Finally, note that this first order approximation is not required when normalization of blackbody or normalization of powerlaw varies.

Considering the above procedure, our model fractional rms amplitude has five parameters: $N_{\mathrm{BB}}, T_{\mathrm{BB}}, N_{\mathrm{PL}}, \alpha_{\mathrm{PL}}$ and $A$, the last one is the fractional peak amplitude of one of the first four parameters, which is fluctuating (e.g., $\frac{T_{\mathrm{BB} 1}}{T_{\mathrm{BB} 0}}$ in the case discussed above for the varying blackbody temperature). While computing the models, we consider following ranges of our parameters: $N_{\mathrm{BB}}=0.5-50, T_{\mathrm{BB}}=$ $0.3-3.0 \mathrm{keV}, N_{\mathrm{PL}}=0.5-50$ photons cm $\mathrm{cm}^{-2} \mathrm{~s}^{-1} \mathrm{keV}^{-1}$ (at $1 \mathrm{keV}), \alpha_{\mathrm{PL}}=1.5-3.5$ and $A=0.001-0.9$. We note that 
the formula for blackbody photon count rate per $\mathrm{cm}^{2}$ per $\mathrm{keV}$ is $\left(N_{\mathrm{BB}} \times 1.0344 \times 10^{-3} \times E^{2}\right) /\left(\exp \left(E / T_{\mathrm{BB}}\right)-1\right)$, while that for powerlaw photon count rate per $\mathrm{cm}^{2}$ per $\mathrm{keV}$ is $N_{\mathrm{PL}} E^{-\alpha_{\mathrm{PL}}}$, with energy $E$ in $\mathrm{keV}$. The best-fit parameter values from our fitting of a few $4 \mathrm{U}$ 1728-34 spectra (see earlier in this section), and from Tarana et al. (2011) and Seifina and Titarchuk (2011) are within the above ranges.

For a set of five model-parameter values, we numerically compute the fractional rms amplitude, as outlined above, systematically in each of the observed energy ranges for the sinusoidal oscillation in time of each of $N_{\mathrm{BB}}, T_{\mathrm{BB}}, N_{\mathrm{PL}}$ and $\alpha_{\mathrm{PL}}$. For each of these four cases, we produce many 'fractional rms amplitude versus energy' model curves for various sets of parameter values, in order to thoroughly explore if some curves for a given case can reasonably describe the data. We show some examples of these model curves in Figs. 5, 6, 7)and 8, In each of these figures, for the variation of a single model parameter, we show the example curves for various sets of parameter values (mentioned in the figure-caption). Therefore, these figures give an idea about the nature of energy-dependence of fractional rms amplitudes for various parameter values for the sinusoidal oscillation in time of a given parameter. The blue dashed curve in each figure appears to be the closest to the data among our computed model curves. These figures show that the sinusoidal oscillation of a blackbody parameter describes the data well, and hence the observed energydependence of fractional rms amplitude is expected. We note that testing this was the main aim of modeling reported in this paper, as mentioned in the first paragraph of this section. Figs. 5, 6, 77 and 8] also suggest that the oscillation of a single blackbody parameter (temperature or normalization) describes the data better than the variation of a single powerlaw parameter (index or normalization). We further note that the small value of $A$ corresponding to the blue dashed curve of Fig. [5] satisfies the assumption of small fluctuation of the blackbody temperature, as mentioned earlier in this section.

\section{DISCUSSION}

In this paper, we report the decrease of fractional rms amplitude of lower $\mathrm{kHz}$ QPOs at higher energies, for the first time to the best of our knowledge. As the count rate of neutron star LMXBs decreases at higher energies, it is difficult to reliably measure the fractional rms amplitude with $R X T E$ PCA (the only instrument capable of detecting $\mathrm{kHz}$ QPOs) due to the lack of sufficient S/N. Sometimes because of this, the previous studies, to the best of our knowledge, have concluded that the fractional rms amplitude either goes on increasing with energies, or first increases and then saturates. In order to improve the $\mathrm{S} / \mathrm{N}$, we combine tens of lower $\mathrm{kHz}$ QPOs from $4 \mathrm{U} 1728-34$, and also use the standard shift-and-add technique $(\S 2)$. The net count rate of our highest energy range $(10.63-20.62$ $\mathrm{keV}$ ) is also larger than that in some other energy ranges. Since the $10.63-20.62 \mathrm{keV}$ range does not have any clear $\mathrm{kHz}$ QPO peak, the decrease of fractional rms amplitude at higher energies should be real, and not a result of low S/N (see Figs. 1 and 2). Note that the small uncertainties in the channel-energy conversion cannot make the lower $\mathrm{kHz}$ QPO disappear at higher energies, and hence cannot produce the decrease. Moreover, the gradual and systematic change of fractional rms amplitude at higher energies for overlapping energy ranges (Figs. 1, 2, 3) strongly supports the decrease of this amplitude of lower $\mathrm{kHz}$ QPOs.

The energy dependence of fractional rms amplitude can be very useful to probe the physical origin of $\mathrm{kHz}$ QPOs (see $\S$ 1), and hence our finding is important. Therefore, we use a two-component (blackbody+powerlaw) spectral model to gain insights (see $\S 4$ for details). We explore the shape and fractional rms amplitude values of many model curves, examples of which have been shown in Figs. 5, 6, 7 and 8 . These curves suggest that (1) the reported energy behavior of lower $\mathrm{kHz}$ QPO fractional rms amplitude can be explained with simple models; and (2) it is likely that the fluctuation in a blackbody-like component primarily causes the lower $\mathrm{kHz}$ QPOs. However, we consider the fluctuation of only one model parameter at a time (§ 4), and hence we cannot study the effects of a simultaneous fluctuation of the two powerlaw parameters. We also note that the non-dependence of Q-value on energy ( $\S 2$ 2) may imply that the lifetime of the oscillations is independent of photon energies. This may suggest that the lower $\mathrm{kHz}$ QPOs in various energy ranges originate from the same location, and hence from the same spectral component. But we cannot constrain the location of either the blackbody or the powerlaw component from our study. The blackbody could originate either from the boundary layer or the disk (Tarana et al. 2011). Similarly, the corona, which plausibly gives rise to the powerlaw, could be centrally located near the neutron star or a cover on the accretion disk.

With our new finding, an important question to ask would be whether the fractional rms amplitude of lower $\mathrm{kHz}$ QPOs decreases with energy (at higher energies) for other neutron star LMXBs as well. Gilfanov et al. (2003) reported that $4 \mathrm{U} 1608-52$ did not show any decrease in fractional rms amplitudes at energies as high as $20 \mathrm{keV}$, although at the high energy end the error in fractional rms amplitude was quite high. A larger (than PCA) area at higher energies may be required (1) to detect a plausible decrease which happens at higher energies than that for $4 \mathrm{U} 1728-34 ;(2)$ and to reduce the errors in fractional $\mathrm{rms}$ amplitudes, while increasing the number of data points, in order to perform a better modeling. The upcoming space missions (e.g., ASTROSAT) having sufficient time resolution and much higher (than PCA) area in $20-50 \mathrm{keV}$, will be useful for this purpose.

We thank Manoneeta Chakraborty and H. M. Antia for technical discussions. A.M. acknowledges Ranjeev Misra for a discussion on the models and thanks M. Coleman Miller for useful general discussions on $\mathrm{kHz}$ QPOs. We also thank an anonymous referee for very constructive comments, which significantly improved the paper. 


\section{REFERENCES}

Abramowicz, M. A., \& Kluźniak, W. 2001, A\&A, 374, L19

Alpar, M. A., \& Psaltis, D. 2008, MNRAS, 391, 1472

Barret, D. 2001, Advances in Space Research, 28, 307

Barret, D., Kluniak, W., Olive, J.-F., Paltani, S. \& Skinner, G. K. 2005a, MNRAS, 357, 1288

Barret, D., Olive, J.-F., \& Miller, M. C. 2005b, MNRAS, 361, 855

Barret, D., Olive, J.-F., \& Miller, M. C. 2006, MNRAS, 370, 1140

Bhattacharyya, S. 2010, Advances in Space Research, 45, 949

Cabanac, C., Henri, G., Petrucci, P. -O., Malzac, J., Ferreira, J., \& Belloni, T. M. 2010, 404, 738

Christian, D. J., \& Swank, J. H. 1997, ApJ, 109, 177

Church, M. J. \& Balucińska-Church, M. 2001, A\&A, 369, 915

Di Salvo, T., Méndez, M., van der Klis, M., Ford, E., \& Robba, N. R. 2001, ApJ, 546, 1107

Ford, E. C., \& van der Klis, M. 1998, ApJ, 506, L39

Gilfanov, M., Revnivtsev, M., \& Molkov, S. 2003, A\&A, 410, 217

Gierliński, M., \& Zdziarski, A. A. 2005, MNRAS, 363, 1349

Kato, S. 2011, Publ. Astron. Soc. Japan 63, 861

Kluzniak, W., \& Abramowicz, M. A. 2001, Acta Physica Polonica B, 32, 3605

Lamb, F. K., \& Miller, M. C. 2003, submitted (astro-ph/0308179)

Leahy, D. A., Darbro, W., Elsner, R. F., Weisskopf, M. C. Sutherland, P. G., Kahn, S., \& Grindlay, J. E., 1983, ApJ, 266, 160

Lee, W. H., Abramowicz, M. A., \& Kluźniak, W. 2004, ApJ, 603, L93

Lee, H. C., \& Miller, G. S. 1998, MNRAS, 299, 479

Lin, Y. F., Boutelier, M., Barret, D., \& Zhang, S. N. 2011, ApJ, 726,74

Lin, D., Remillard, R. A., \& Homan, J. 2007, ApJ, 667, 1073

Lin, D., Remillard, R. A., \& Homan, J. 2010, ApJ, 719, 1350

Maccarone, T. J., \& Coppi, P. S. 2003, A\&A, 399, 1151

Maitra, D., \& Bailyn, C. D. 2004, ApJ, 608, 444

Méndez, M. 2006, MNRAS, 371, 1925

Méndez, M., \& Belloni, T. 2007, MNRAS, 381, 790

Méndez, M., \& van der Klis, M. 1999, ApJ, 517, L51

Méndez, M., van der Klis, M., \& Ford, E. C. 2001, ApJ, 561, 1016

Méndez, M., van der Klis, M., van Paradijs, J., Lewin, W. H. G., Vaughan, B. A., Kuulkers, E., Zhang, W., Lamb, F. K. \& Psaltis, D. 1998, ApJ, 494, L65
Miller, M. C., Lamb, F. K., \& Psaltis, D. 1998, ApJ, 508, 791

Mitsuda, K., Inoue, H., Nakamura, N., \& Tanaka, Y. 1989, PASJ, 41,97

Mitsuda, K., \& Dotani, T. 1989, PASJ, 41, 557

Mukherjee, A., \& Bhattacharyya, S. 2011, MNRAS, 411, 2717

Mukherjee, A., \& Bhattacharyya, S. 2011, ApJ, 730, L32

Mukhopadhyay, B. 2009, ApJ, 694, 387

Muno, M. P., Ozel, F., \& Chakrabarty, D. 2002, ApJ, 581, 550

Olive, J. F., Barret, D., \& Gierlński, M. 2003, 583, 416

Pétri, J. 2011, Astrophysics and Space Science, 331, 555

Psaltis, D. 2008, Living Reviews in Relativity, 11, 9

Romanova, M. M., \& Kulkarni, A. K. 2009, MNRAS, 398, 1105

Seifina, E., \& Titarchuk, L. 2011, ApJ, 738, 128

Stella, L., \& Vietri, M. 1998, ApJ, 492, L59

Stella, L., \& Vietri, M. 1999, Physical Review Letters, 82, 17

Strohmayer, T. E., Zhang, W., Swank, J. H., Smale, A., Titarchuk, L., Day, C., \& Lee, U. 1996, ApJ, 469, L9

Stuchlík, Z., Kotrlová, A., \& T:or:ok, G. 2011, A\&A, 525, 82

Tarana, A., Belloni, T., Bazzano, A., Mndez, M., \& Ubertini, P. 2011, MNRAS, 416, 873

Török, G. 2009, A\&A, 497, 661

van der Klis, M. 1989, Eds. H. Ögelman and E.P.J. van den Heuvel, (Kluwer Academic Publishers: Boston), 27

van der Klis, M. 2006, Compact stellar X-ray sources, 39

van der Klis, M., Swank, J. H., \& Zhang, W. 1996, ApJ, 469, L1

van der Klis, M., Swank, J. H., Zhang, W., Jahoda, K., Morgan, E. H., Lewin, W. H. G., Vaughan, B., \& van Paradijs, J. 1996, ApJ, 469, L1

Wang, J., Zhang, C. M., Zhao, Y. H., Lin, Y. F., Yin, H. X., \& Song, L. M. 2011, A\&A, 528, 126

White, N. E., Stella, L., \& Parmar, A. N. 1988, ApJ, 324, 363

Wijnands, R. 2001, Advances Space Res., 28, 469

Wijnands, R., van der Klis, M., Homan, J., Chakrabarty, D., Markwardt, C. B., \& Morgan, E. H. 2003, Nature, 424, 44

Zdziarski, A. A., Gierliński, M., Rao, A. R., Vadawale, S. V., \& Mikołajewska, J. 2005, MNRAS, 360, 825

Zhang, C. 2004, A\&A, 423, 401

Zhang, C. M., Yin, H. X., Zhao, Y. H., Zhang, F., \& Song, L. M. 2006, MNRAS, 366, 1373 

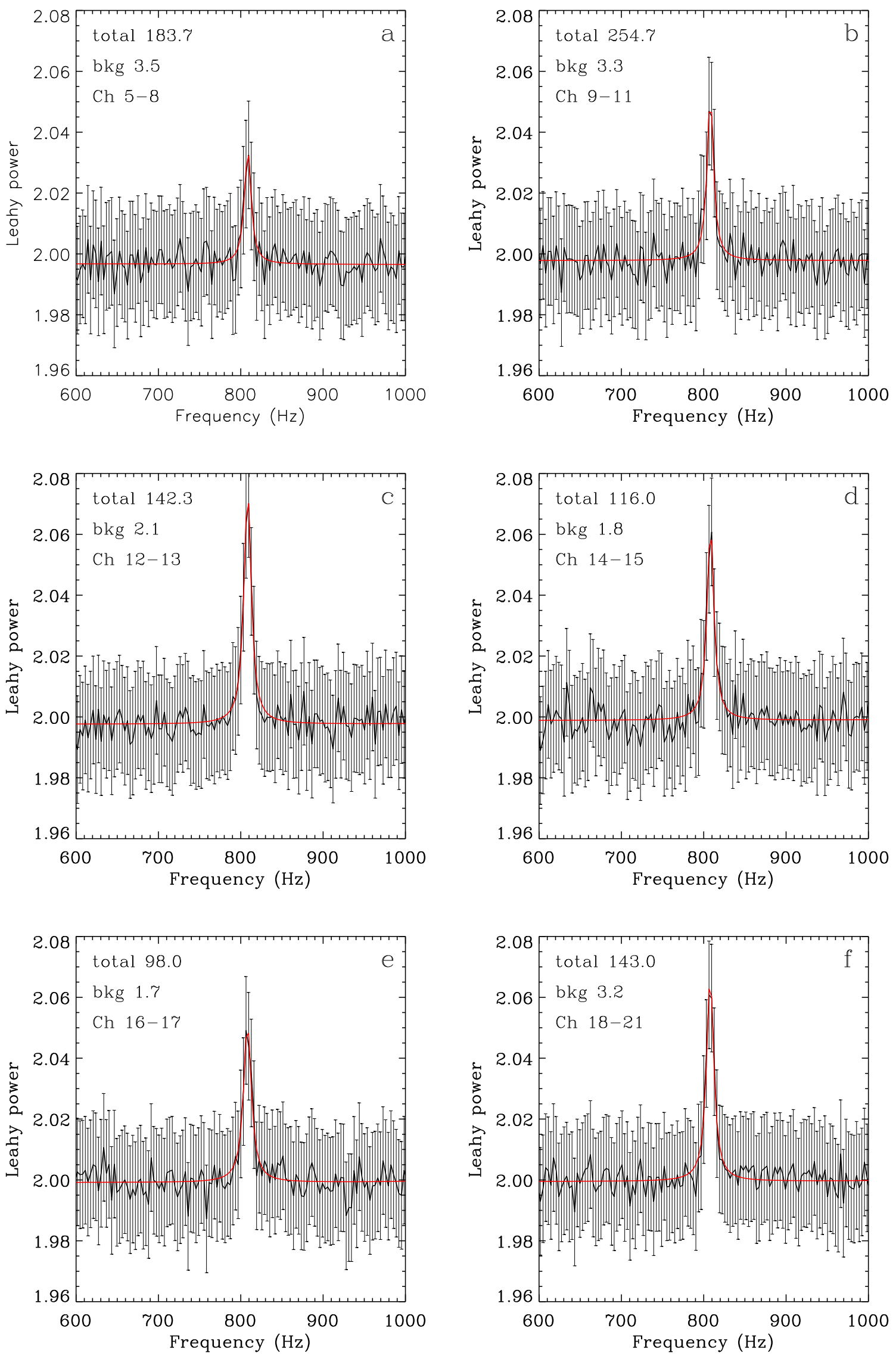

FIG. 1. - Leahy-normalized power density spectra (PDS; with $1 \sigma$ errors) of $4 \mathrm{U} 1728-34$ after using the shift-and-add technique for each of the energy ranges 2.06-3.68 keV (panel a), 3.68-4.90 keV (panel b), 4.90-5.71 keV (panel c), 5.71-6.53 keV (panel $d$ ), 6.53-7.35 keV (panel $e$ ) and 7.35-8.98 keV (panel $f$; see $\S 2$ ). In each panel, the average total count rate (i.e. without background subtraction) used to compute the PDS is denoted with 'total', the average background count rate is denoted with 'bkg', and the PCA absolute channel range is denoted with 'Ch'. Furthermore, the frequency resolution and exposure in each panel are $3.2 \mathrm{~Hz}$ and $69.2 \mathrm{ks}$, respectively. A lower kHz QPO peak is 

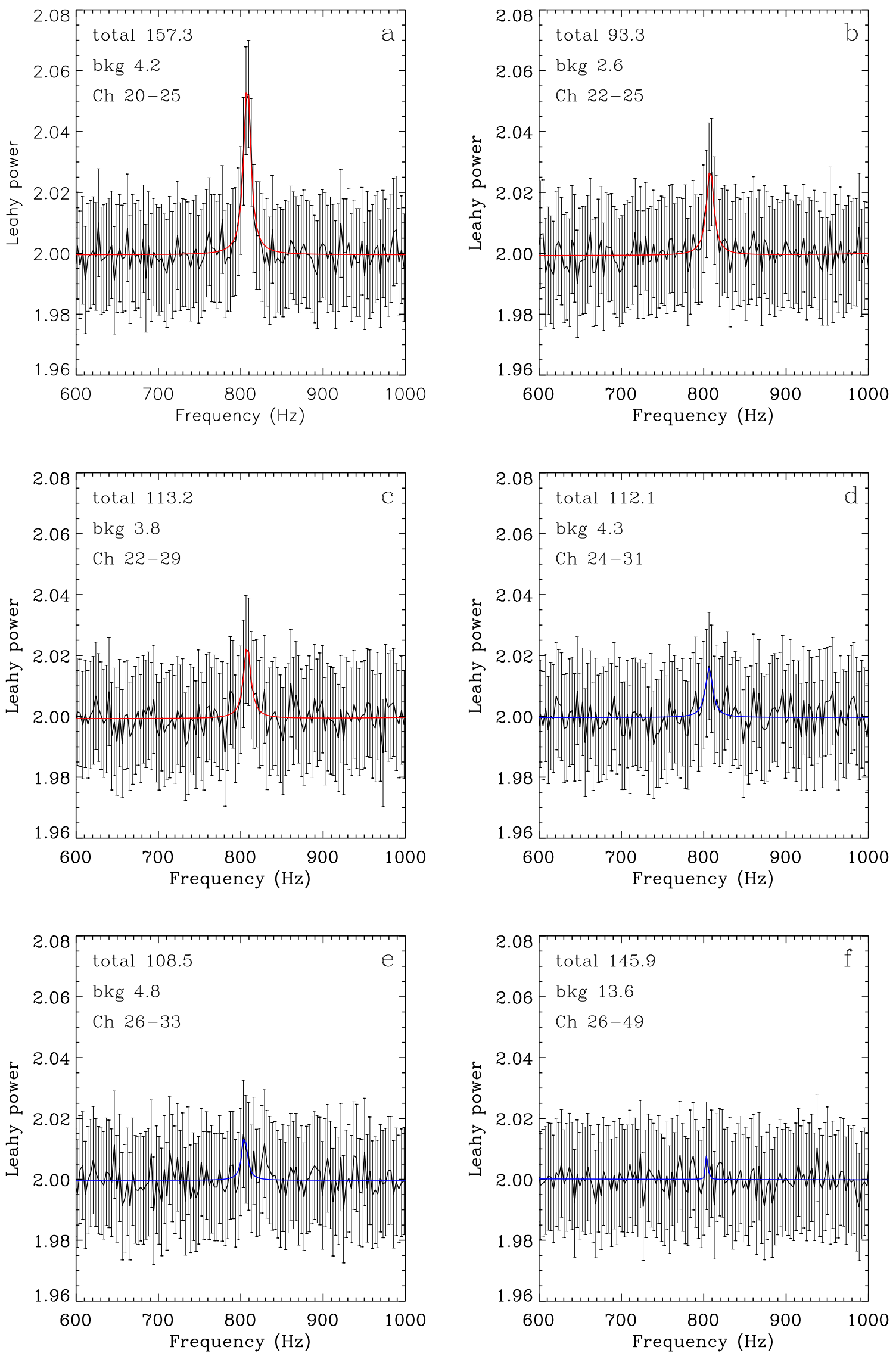

FIG. 2.- Same as Fig. 1 but for the energy ranges $8.17-10.63 \mathrm{keV}$ (panel $a$ ), 8.98-10.63 keV (panel $b$ ), 8.98-12.28 keV (panel $c$ ), 9.81-13.11 $\mathrm{keV}$ (panel $d$ ), 10.63-13.93 keV (panel $e$ ) and 10.63-20.62 keV(panel $f$; see $\S$ 2). In the panels $d$, $e$ and $f$, the lower $\mathrm{kHz}$ QPO peak is not clearly seen, and hence the best-fit model curves in these panels are marked with a different color (blue). For each of these three energy ranges, an upper limit to the lower $\mathrm{kHz}$ QPO fractional rms amplitude is computed (see $\S$ 3). 


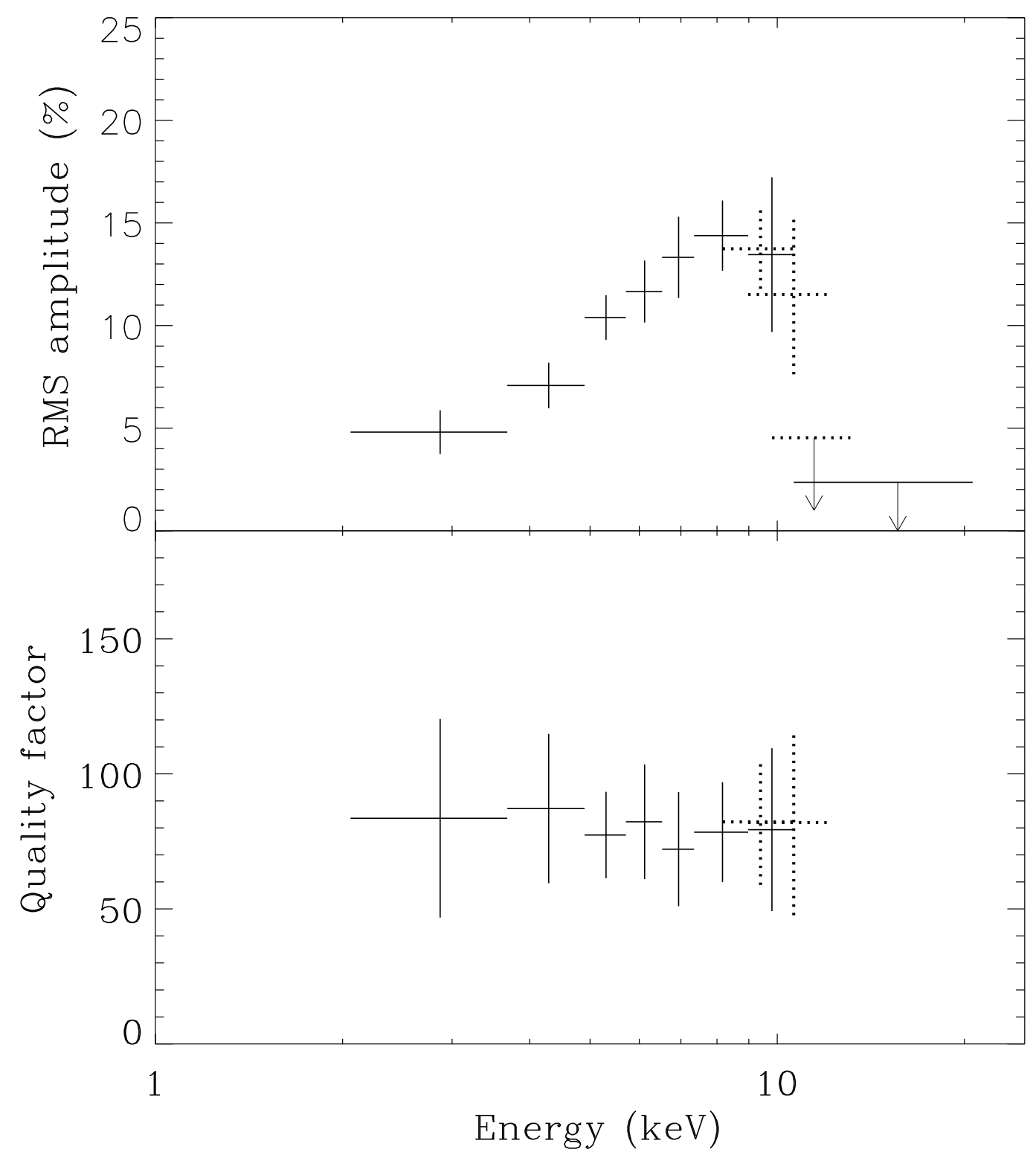

FIG. 3.- Energy dependence of the background corrected fractional rms amplitude and the Q-value of the lower kHz QPO from $4 \mathrm{U} 1728-34$. In each panel, solid horizontal lines denote a set of energy ranges which are mutually non-overlapping, and energy ranges denoted with dotted horizontal lines overlap with some ranges denoted with solid lines. Upper panel: rms amplitude (with $1 \sigma$ error or $1 \sigma$ upper limit) versus energy with ranges 2.06-3.68 keV (Fig. 1] panel a), 3.68-4.90 keV (Fig. 1 panel b), 4.90-5.71 keV (Fig. 1 panel c), $5.71-6.53 \mathrm{keV}$ (Fig. 1 ] panel d), 6.53-7.35 keV (Fig. 1 panel e), 7.35-8.98 keV (Fig. 1 panel f), 8.17-10.63 keV (Fig. 2 panel a), 8.98-10.63 keV (Fig. 2 panel b), 8.98-12.28 keV (Fig. 22 panel c), 9.81-13.11 keV (Fig. 22 panel d) and 10.63-20.62 keV (Fig. 2] panel f). This panel shows that the rms amplitude clearly and gradually decreases at higher energies. Lower panel: Q-value (corrected for frequency drift with shift-and-add technique for $400 \mathrm{~s}$ segments; with $1 \sigma$ error) versus energy with ranges $2.06-3.68 \mathrm{keV}$ (Fig. 1 panel a), 3.68-4.90 keV (Fig. [1 panel b), 4.90-5.71 keV (Fig. 1] panel c), 5.71-6.53 keV (Fig. 1] panel d), 6.53-7.35 keV (Fig. 1] panel e), 7.35-8.98 keV (Fig. 1] panel f), 8.17-10.63 keV (Fig. 22 panel a), 8.98-10.63 keV (Fig. 2] panel b) and 8.98-12.28 keV (Fig. 2] panel c). This panel shows that the Q-value does not significantly depend on energy (see $\S 2$ and 3 . 


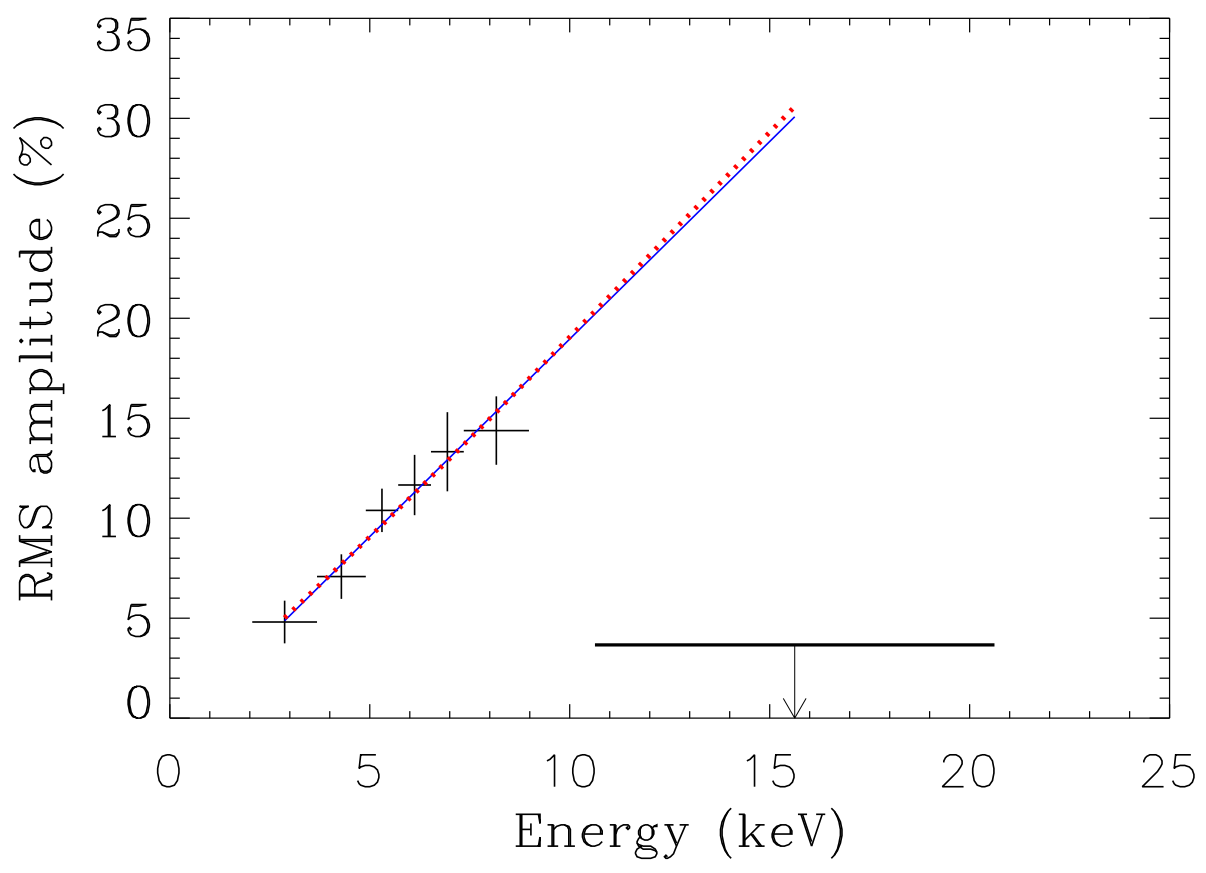

Fig. 4. - Energy dependence of the background corrected fractional rms amplitude of the lower kHz QPO from 4U 1728-34. The first six data points (with $1 \sigma$ errors), which are same as the first six data points of the upper panel of Fig. 3 are fitted with a linear (solid blue line) and a powerlaw model (dotted red line). Both the best-fit models (extrapolated) are shown. The last data point (for the same energy range as that of the last data point of the upper panel of Fig. 31) gives the $3 \sigma$ upper limit of fractional rms amplitude. This figure shows that the increasing trend of amplitude at lower energies cannot explain the last data point. 


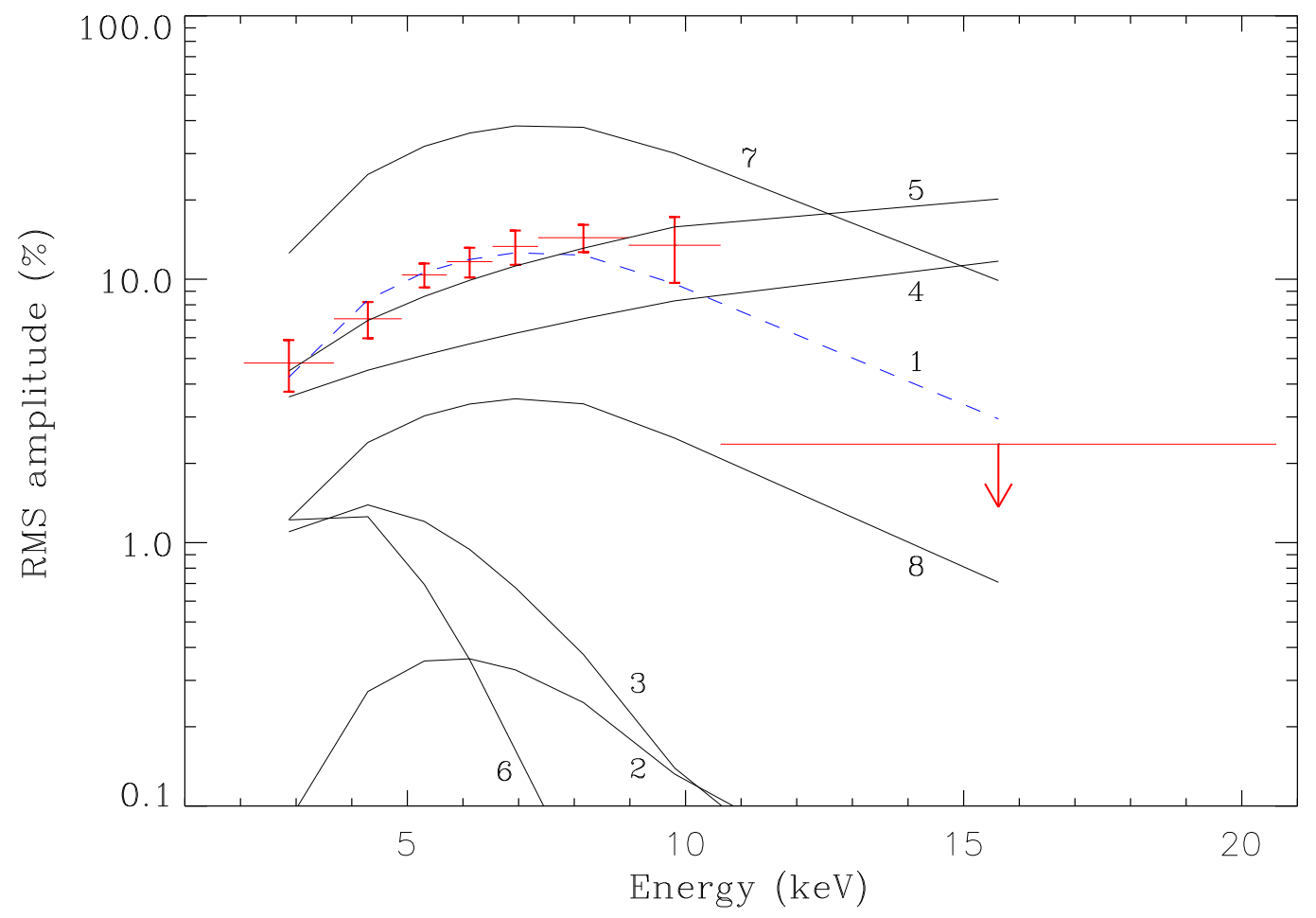

FIG. 5.- The lower $\mathrm{kHz}$ QPO fractional (in \%) rms amplitude vs. energy. The red points are the same non-overlapping data points of the upper panel of Fig. 3 ( $\S 2$ and 3). The curves are models for sinusoidal fluctuation of the blackbody temperature (see $\S$ 4). The parameter $\left(N_{\mathrm{PL}}, \alpha_{\mathrm{PL}}, N_{\mathrm{BB}}, T_{\mathrm{BB}}\right.$ and $A$ ) values for various model curves are as follows in the same sequence (for units see $\S 4$. 1: [0.5, 3.5, 50.0, 0.89, $0.034] ; 2:[0.5,3.5,0.5,0.88,0.034] ; 3:[0.5,1.5,50.0,0.88,0.034] ; 4:[0.5,3.5,50.0,3.0,0.034] ; 5:[0.5,3.5,50.0,1.5,0.034] ; 6:[0.5,3.5$, $50.0,0.5,0.034] ; 7:[0.5,3.5,50.0,0.88,0.1] ; 8:[0.5,3.5,50.0,0.88,0.01]$. The blue dashed curve (curve 1$)$ appears to be the closest to the data among our computed model curves for blackbody temperature fluctuation (see $\S$ 4). This figure shows that blackbody temperature fluctuation could reproduce the observed energy dependence of the lower $\mathrm{kHz}$ QPO fractional rms amplitude ( $(4)$. 


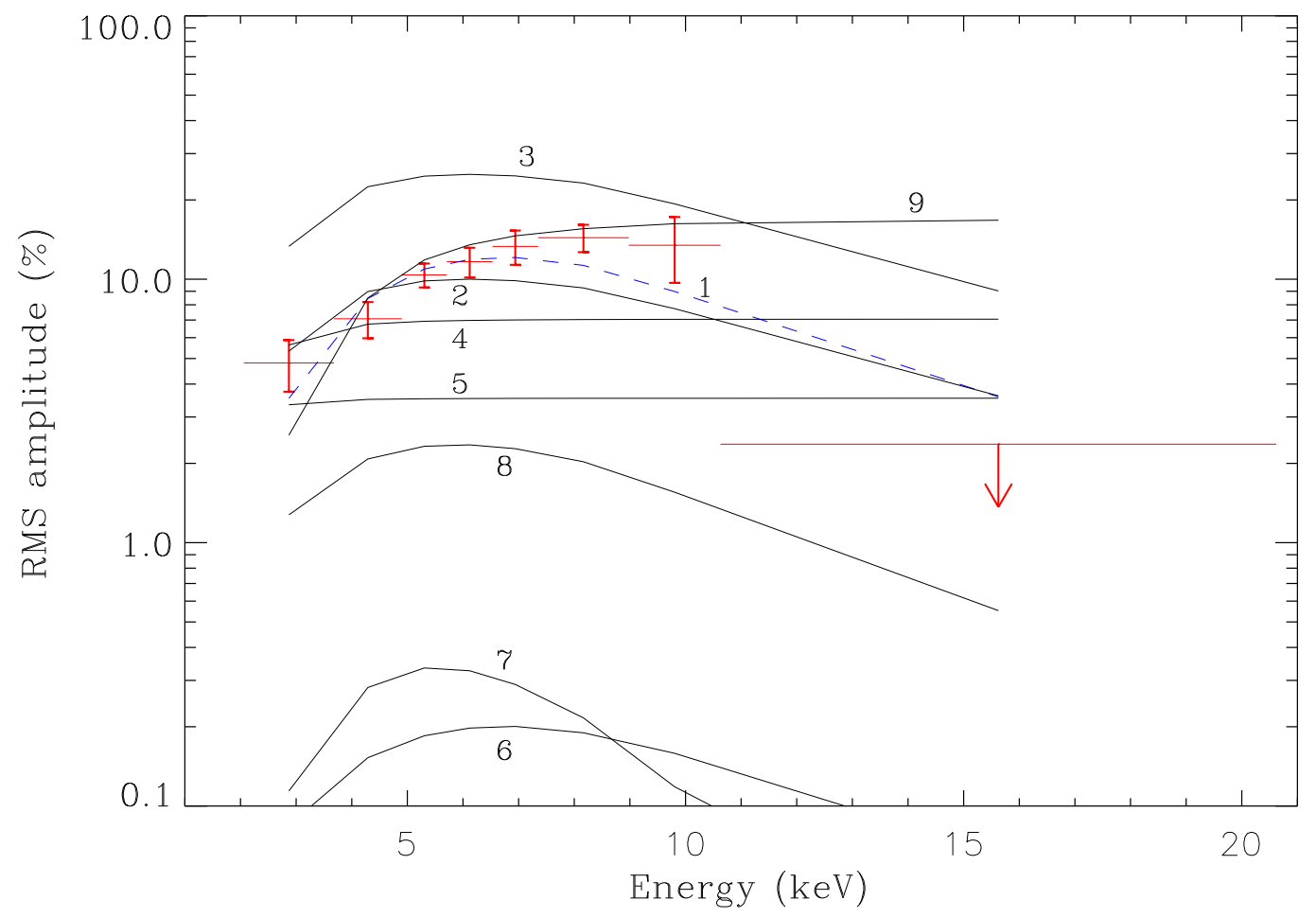

FIG. 6.- Similar to Fig. 5 but the curves are models for sinusoidal fluctuation of the blackbody normalization (see $\S$ 4). The parameter $\left(N_{\mathrm{PL}}, \alpha_{\mathrm{PL}}, N_{\mathrm{BB}}, T_{\mathrm{BB}}\right.$ and $A$ ) values for various model curves are as follows in the same sequence (for units see $\S$ 4). 1: [3.69, 2.79, 10.37, $1.42,0.9] ; 2:[0.5,3.5,13.8,1.11,0.2] ; 3:[0.5,3.5,13.8,1.11,0.5] ; 4:[0.5,3.5,12.4,3.0,0.1] ; 5:[0.5,3.5,50.0,3.0,0.05] ; 6:[10.0,1.5,10.0$, $2.0,0.1] ; 7:[10.0,3.5,10.0,1.0,0.1] ; 8:[0.5,2.0,10.0,1.5,0.1] ; 9:[50.0,3.5,50.0,3.0,0.25]$. The blue dashed curve (curve 1$)$ appears to be the closest to the data among our computed model curves for blackbody normalization fluctuation (see $\S$ 4). This figure shows that blackbody normalization fluctuation could reproduce the observed energy dependence of the lower $\mathrm{kHz}$ QPO fractional rms amplitude, although for a high value of $A(\S 4)$. 


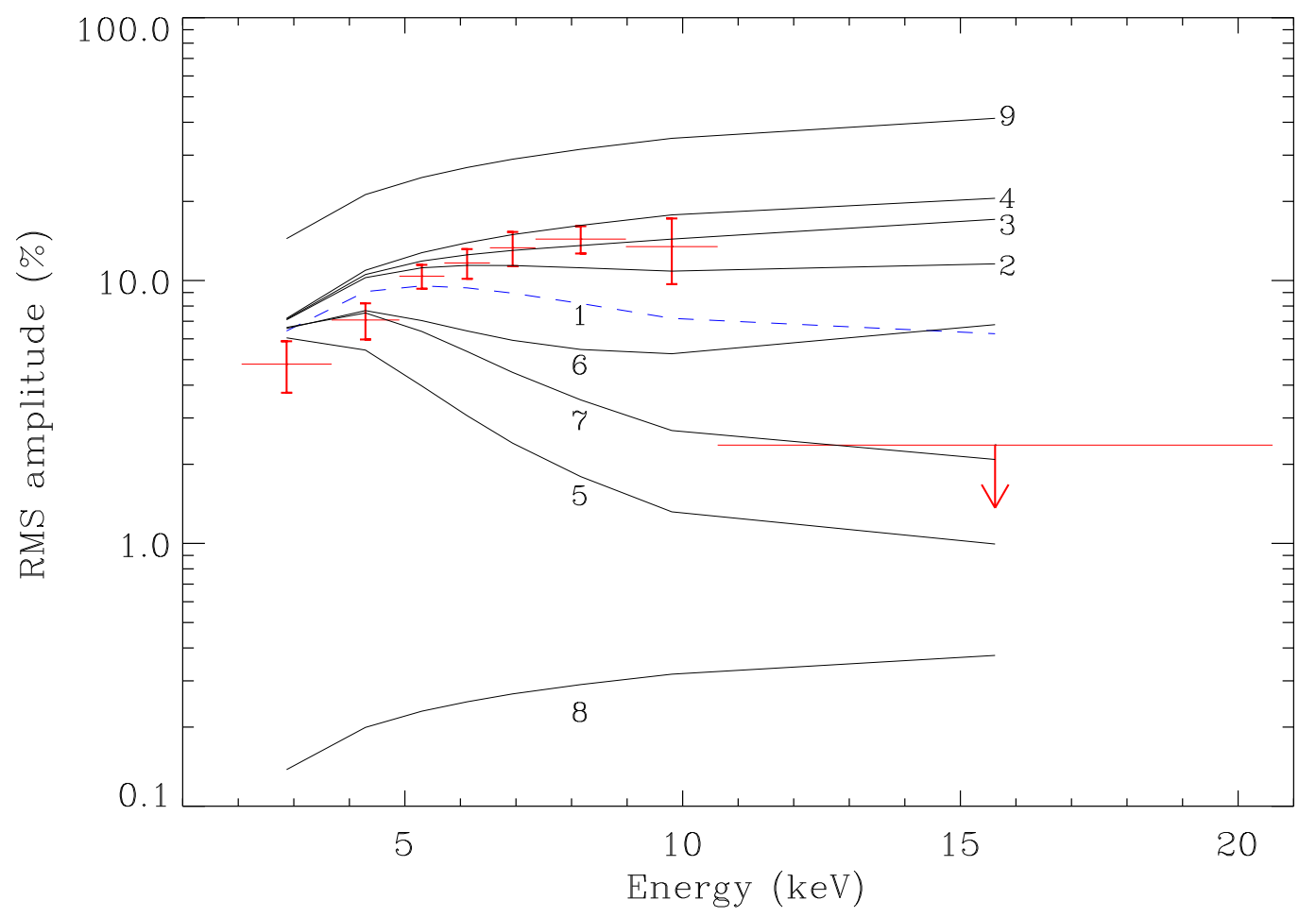

Fig. 7. - Similar to Fig. 5 but the curves are models for sinusoidal fluctuation of the powerlaw index (see $\S 4$ ). The parameter $\left(N_{\mathrm{PL}}, \alpha_{\mathrm{PL}}\right.$, $N_{\mathrm{BB}}, T_{\mathrm{BB}}$ and $A$ ) values for various model curves are as follows in the same sequence (for units see $\S$ 4). 1: [18.55, 3.5, 2.07, 3.0, 0.027]; 2: $[45.0,3.5,5.0,2.5,0.03] ; 3:[45.0,3.5,5.0,2.0,0.03] ; 4:[45.0,3.5,5.0,1.0,0.03] ; 5:[45.0,3.5,50.0,3.0,0.03] ; 6:[45.0,3.5,50.0,2.0,0.03] ; 7$ : $[10.0,3.5,5.0,3.0,0.03] ; 8:[10.0,2.0,10.0,1.5,0.001] ; 9:[10.0,2.0,10.0,1.5,0.1]$. The blue dashed curve (curve 1) appears to be the closest to the data among our computed model curves for powerlaw index fluctuation (see $\S$ 4). This figure shows that powerlaw index fluctuation plausibly could not reproduce the observed energy dependence of the lower kHz QPO fractional rms amplitude (see $\S$ 4). 


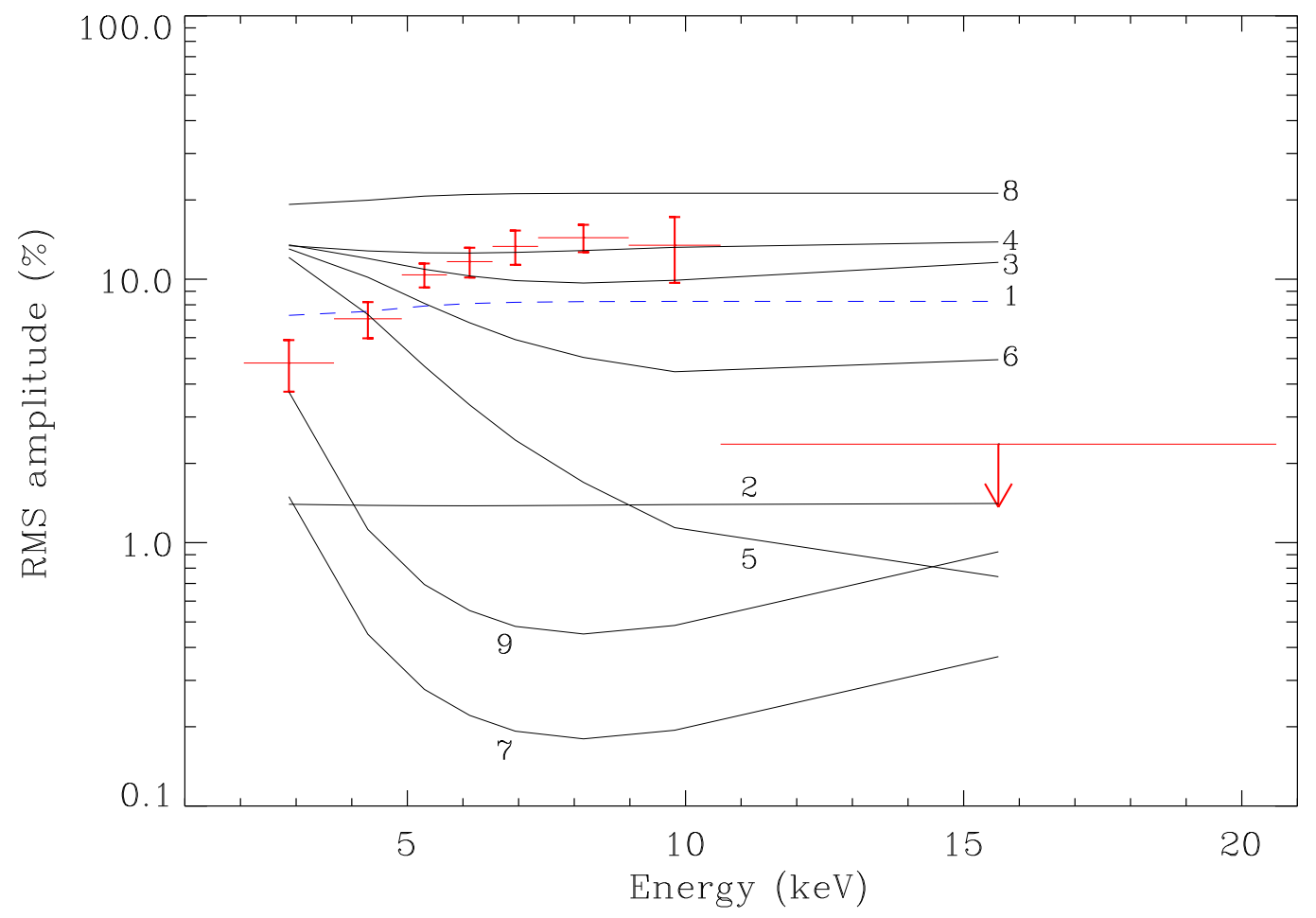

FIG. 8. - Similar to Fig. 5 but the curves are models for sinusoidal fluctuation of the powerlaw normalization (see $\S 4$ ). The parameter $\left(N_{\mathrm{PL}}, \alpha_{\mathrm{PL}}, N_{\mathrm{BB}}, T_{\mathrm{BB}}\right.$ and $\left.A\right)$ values for various model curves are as follows in the same sequence (for units see $\S 4$ ). 1: $[0.5,3.5,50.0,0.52$, $0.117] ; 2:[10.0,2.0,10.0,1.5,0.02] ; 3:[10.0,3.5,10.0,1.5,0.2] ; 4:[10.0,2.0,50.0,1.5,0.2] ; 5:[50.0,3.5,50.0,3.0,0.2] ; 6:[0.5,3.5,0.5$, $2.0,0.2] ; 7:[0.5,3.5,50.0,1.5,0.12] ; 8:[0.5,3.5,50.0,0.5,0.3] ; 9:[0.5,3.5,50.0,1.5,0.3]$. The blue dashed curve (curve 1 ) appears to be the closest to the data among our computed model curves for powerlaw normalization fluctuation (see $\S$ 4). This figure shows that powerlaw normalization fluctuation plausibly could not reproduce the observed energy dependence of the lower $\mathrm{kHz}$ QPO fractional rms amplitude (see $\S$ (4). 